\title{
Pulmonary tumours in the Netherlands: focus on temporal trends in histology and stage and on rare tumours
}

\author{
W K de Jong, ${ }^{1}$ M Schaapveld, ${ }^{2}$ J L G Blaauwgeers, ${ }^{3}$ H J M Groen ${ }^{1}$
}

${ }^{1}$ Department of Pulmonology, University Medical Center Groningen, University of Groningen, The Netherlands; ${ }^{2}$ Comprehensive Cancer Center Northern Netherlands, Groningen, The Netherlands;

${ }^{3}$ Department of Pathology, Onze Lieve Vrouwe Gasthuis,

Amsterdam, The Netherlands

Correspondence to: Dr W K de Jong, Department of Pulmonology, University Medical Center Groningen, P 0 Box 30.001, NL-9700 RB Groningen, The Netherlands; w.k.de.jong@ int.umcg.nl

Received 20 December 2007

Accepted 8 July 2008

Published Online First

4 August 2008

\begin{abstract}
Background: Recent temporal trends in histology and stage of pulmonary tumours in the Netherlands were studied. The incidence of rare pulmonary tumours was determined.

Methods: All tumours originating from the trachea, bronchus and lung recorded in the Netherlands Cancer Registry were included. Based on ICD-0 morphology codes, five major subgroups were constructed: squamous carcinoma (SC), adenocarcinoma (AC), large cell (undifferentiated) carcinoma (LC), small cell lung cancer (SCLC) and other (including uncommon tumours).
\end{abstract}

Results: Between 1989 and 2003, 134894 tumours were diagnosed. In men the age-adjusted incidence of SC and SCLC decreased, AC remained stable and LC increased. In women the incidence of all subgroups increased. Since 1996, a stage shift was observed with fewer patients in stage I and more patients in stage IV at diagnosis. This stage shift occurred equally in SC, AC and LC. In SC, fewer patients presented with stage IV disease than in AC and LC ( $25 \%$ vs $44 \%$ and $49 \%$ in 2003 , respectively). The incidence of adenosquamous carcinoma decreased from 0.6 to $0.29 / 100000(p<0.001)$. The incidence of carcinoid tumours, sarcomatoid carcinomas and primary pulmonary sarcomas remained stable $(0.44$, 0.17 and 0.08/100 000, respectively).

Conclusion: The incidence of smoking-related tumours decreased in men (especially SC and SCLC) and increased in women (all subgroups). More patients presented with stage IV disease. The incidence of non-smoking-related uncommon tumours remained constant.

Lung cancer is the most common thoracic tumour, accounting for more than 350000 deaths annually in Europe ${ }^{1}$ and 160000 in the USA. ${ }^{2}$ In Europe the age-adjusted incidence varies between 45 and 80/ 100000 for men (highest in Eastern Europe) and between 5 and 20/100 000 for women. ${ }^{13}$ The ageadjusted incidence of lung cancer among men in the USA is $50-80 / 100000$ and $20-55 / 100000$ in women. ${ }^{23}$

Lung cancer is generally divided into small cell lung cancer (SCLC) and non-small cell lung cancer (NSCLC), the latter being subdivided into squamous carcinoma (SC), adenocarcinoma (AC) and large cell (undifferentiated) carcinoma (LC). The major cause of lung cancer is tobacco smoking, and the association between lung cancer and smoking habits is firmly defined. ${ }^{4}$

The characteristics of lung cancer are changing worldwide. ${ }^{5}$ Changes in incidence are associated with changes in smoking habits. The proportion of men who smoke has declined since 1960 in most countries, including the Netherlands, ${ }^{67}$ but has increased in Eastern Europe. ${ }^{1}$ The proportion of women who smoke increased until the 1970s and thereafter declined in most countries. Currently, the proportions of men and women who smoke are approaching each other in the Netherlands (36\% and $29 \%$ of all adults, respectively ${ }^{8}$.

Apart from the overall incidence, the relative frequencies of the different histological subtypes of lung cancer also seem to have changed over the last decades. This change could partially be explained by the introduction of the low tar filter cigarette in the 1960s, ${ }^{9}$ because filter cigarettes require deeper inhalation. Deeper inhalation results in a more peripheral particle deposit and therefore a higher probability of the more peripherally occurring AC. In the Netherlands the filter cigarette is still gaining popularity, especially among women. ${ }^{8}$

One could also expect changes in the distribution of disease stages at presentation due to improvements in imaging techniques and more thorough surgical approaches. The occurrence and magnitude of these stage shifts is unknown.

Several other tumours are diagnosed in the lungs with a much lower frequency than NSCLC and SCLC. The most well known of these tumours are carcinoid tumours, sarcomatoid carcinomas and primary pulmonary sarcomas, all deriving from orthotopic pulmonary tissue. The incidence-and especially the changes in incidence-of these rare tumours is not well defined.

The aim of the present study was to examine the temporal trends in histological classification and staging of lung cancer in the Netherlands over a 15year period and to determine the incidence of less common pulmonary tumours.

\section{METHODS}

\section{Netherlands Cancer Registry}

Data were retrieved from the Netherlands Cancer Registry (NCR). The NCR receives data from nine regional cancer registries and has had nationwide coverage since 1989. Completeness of case ascertainment of the NCR is over $98 \% .^{10}$ The database of the NCR includes information on age, sex, year of diagnosis, tumour topography (primary site of origin), tumour morphology according to the International Classifications of Disease-Oncology $(\mathrm{ICD}-\mathrm{O})^{11}$ and disease stage. Information on smoking history was not available.

This study was approved by the NCR scientific committee and all procedures were performed according to NCR privacy regulations. 


\section{Patient selection}

All patients diagnosed between 1 January 1989 and 31 December 2003 with a tumour located in the trachea, bronchus or lung (topography codes C.33 and C.34) were included. Tumours originating in the thymus, heart, mediastinum or the pleura (C.37 and C.38) were not included. For incidence and disease stage evaluations, all tumours were categorised into five categories: squamous cell carcinoma (SC; ICD-O morphology codes 8050-8052, 8070-8078, 8083, 8084, 8094), adenocarcinoma (AC; 8140, 8200, 8230, 8250-8255 (including bronchioalveolar carcinoma), 8260, 8263, 8323, 8333, 8430, 8470, 8480$8481,8490,8550,8570)$, large cell undifferentiated carcinoma (LC; 8010-8014, 8020-8021, 8046, 8082, 8090, 8123, 8246 (poorly differentiated), 8310, 8574; thereby including NSCLC not characterised as AC or SC), small cell lung cancer (SCLC; 8041-8045) and other (remaining morphology codes). This last category encompasses the rarer pulmonary tumours such as adenosquamous carcinoma (8560), carcinoid tumours (82408243, 8245, 8246 (well and moderately differentiated; 8249), sarcomatoid carcinomas (pleomorphic/giant cell/spindle carcinomas) (8022, 8030-8033, 8575, 8980, 8981, 8972), primary pulmonary sarcomas (8800-8921, 8930-8931, 8935, 8936, 8990, 8991, 9040-9044, 9120-9342, 9364-9372, 9540-9581) and tumours with unknown or other morphology codes. Lymphoma and leukaemia are not within the selection.

For NSCLC (SC, AC and LC), disease stage was based on the Tumour-Node-Metastasis (TNM) classification. In the absence of pathological TNM stage, clinical TNM was used. SCLC was staged as limited or extensive disease.

\section{Statistical analysis}

The population at risk for each year was determined from data from Statistics Netherlands. ${ }^{12}$ Incidence rates were calculated per 100000 person years according to sex, histological subtype and year of diagnosis. Incidence rates were age-standardised using the European Standard Population as reference. ${ }^{3}$ Trends were estimated by calculating the Estimated Annual Percentage Change (EAPC) with a 95\% confidence interval (CI). All reported $p$ values are two-sided; the statistical significance level was set at a $p$ value of $<0.05$.

\section{RESULTS}

\section{Patient characteristics}

Between 1989 and 2003, 134984 pulmonary tumours were diagnosed in the Netherlands, of which 30600 (22.7\%) occurred in women. The proportion of women doubled from $15.2 \%$ in 1989 to $32.0 \%$ in 2003 ( $\mathrm{p}<0.001)$. The median age at diagnosis was 68.6 years (IOR 60.9-72.2), 69.4 years for men and 65.0 years for women $(p<0.001)$.

\section{Temporal trends in histological classification}

The total number of pulmonary tumours slightly increased over time (approximately 9000 annually, EAPC $+0.2 \%$, CI $0.0 \%$ to $0.4 \%, p=0.04)$. In men the incidence of all pulmonary tumours together substantially decreased from 109.2/100 000 in 1989 to $71.6 / 100000$ in 2003 (EAPC $-3.1 \%$, CI $-3.4 \%$ to $-2.8 \%$, $\mathrm{p}<0.001)$. This is largely accounted for by the decrease in incidence of SC and SCLC (from 49.0 and 19.5 to 21.2 and 11.2/ 100 000, respectively; $p<0.001$, fig 1). The incidence of SC in men decreased by a striking $57 \%$ over 15 years, and does not seem to have plateaued yet. The incidence of AC remained constant at 15.9/100 000 and the incidence of LC increased slightly from 15.7 to $17.6 / 100000$ ( $p<0.001)$.
In women the incidence of all pulmonary tumours together increased considerably from 16.9 to $30.5 / 100000$ (EAPC +4.3\%, CI $4.0 \%$ to $4.7 \%, p<0.0001)$, with all histological subgroups involved (from 3.8, 4.9, 2.7 and 4.3 to 5.0, 9.5, 5.3 and 4.8/ 100000 for SC, AC, LC and SCLC, respectively (all $\mathrm{p}<0.001$, fig 1).

Interestingly, the number of large cell neuroendocrine carcinomas (morphology codes 8013,8246 poorly differentiated, and 8574 ) showed a steep increase in both sexes (fig 2, EAPC $+38.3 \%$, CI $29.6 \%$ to $47.6 \%, \mathrm{p}<0.0001)$.

Bronchioalveolar carcinoma (categorised among AC) constituted $7.3 \%$ of all AC in men and $10.3 \%$ of all AC in women. The incidence of bronchioalveloar carcinoma was stable in men and increased in women, which is in line with the incidence of all AC.

\section{Temporal trends in stage distribution}

In NSCLC (SC, AC and LC) the percentage of patients with stage I disease at presentation decreased over time in men, women and in both sexes combined (EAPC $-2.7 \%$, CI $-3.4 \%$ to $-2.1 \%, p<0.001$, fig 3). The percentages of stages II and III remained stable at approximately $6 \%(p=0.28)$ and $35 \%$ $(p=0.20)$, respectively. The percentage of patients with stage IV at diagnosis increased (EAPC $+3.9 \%$, CI $3.1 \%$ to $4.6 \%$, $\mathrm{p}<0.001)$. These changes in stage distribution mainly occurred after 1996 and, since approximately the year 2000, stage IV was most commonly diagnosed. Interestingly, women generally presented with higher disease stages, for instance 44\% with stage IV in 2003 compared with only $37 \%$ of men $(p<0.001)$. This difference in staging between men and women is present in all three major histological subtypes. In addition, the proportion of patients with NSCLC of unknown disease stage gradually decreased from $10.3 \%$ to $3.7 \%(p<0.001)$.

These changes in stage distribution are paralleled by changes in stage-specific incidence. The incidence of stage I NSCLC significantly decreased from 12.0 to 7.1/100 000 (men and women combined, EAPC $-4.0 \%$, CI $-4.8 \%$ to $-3.1 \%$, $\mathrm{p}<0.001)$, with a stable incidence of stage II $(2.6 / 100000$ in 2003), a recent decrease in stage III (to 12.9/100 000) and an increase in the incidence of stage IV to $15.2 / 100000$ in 2003 (EAPC $+2.4 \%$, CI $1.7 \%$ to $3.0 \%$, p $<0.001$, fig 4 ).

Stage distribution differed between SC, AC and LC. The most remarkable difference was the percentage of patients with stage IV disease. From 1996 to 2003 the proportion with stage IV disease varied between $40 \%$ and $50 \%$ for AC and LC compared with only $25 \%$ for SC (fig 5). Stage I was more common in SC than in AC and LC. However, the stage shift observed among NSCLC (a decrease in stage I and increase in stage IV after 1996) was observed in all histological subtypes.

After 1998 the percentage of patients with SCLC having extensive disease at presentation gradually increased to $62 \%$ in 2003 (EAPC $+1.7 \%$, CI $1.3 \%$ to $2.2 \%, \mathrm{p}<0.001$ ), whereas the frequency of both limited disease and unknown disease stage decreased (fig 6). This trend was similar for men and women.

\section{Other pulmonary tumours}

The incidence of pulmonary tumours other than SC, AC, LC or SCLC decreased in men (from 9.0 to $5.7 / 100$ 000, EAPC $-2.4 \%$, $\mathrm{CI}-3.2 \%$ to $-1.6 \%, \mathrm{p}<0.001$ ) and increased in women (from 1.4 to $2.3 / 100000$ in 2003 , EAPC $+3.9 \%$, CI $2.7 \%$ to $4.8 \%$, $\mathrm{p}<0.0001)$. The overall majority in the "other" group consisted of unknown or unspecified tumours $77.5 \%$ in men and women combined). Some specific types can be discerned in this "other" group. 

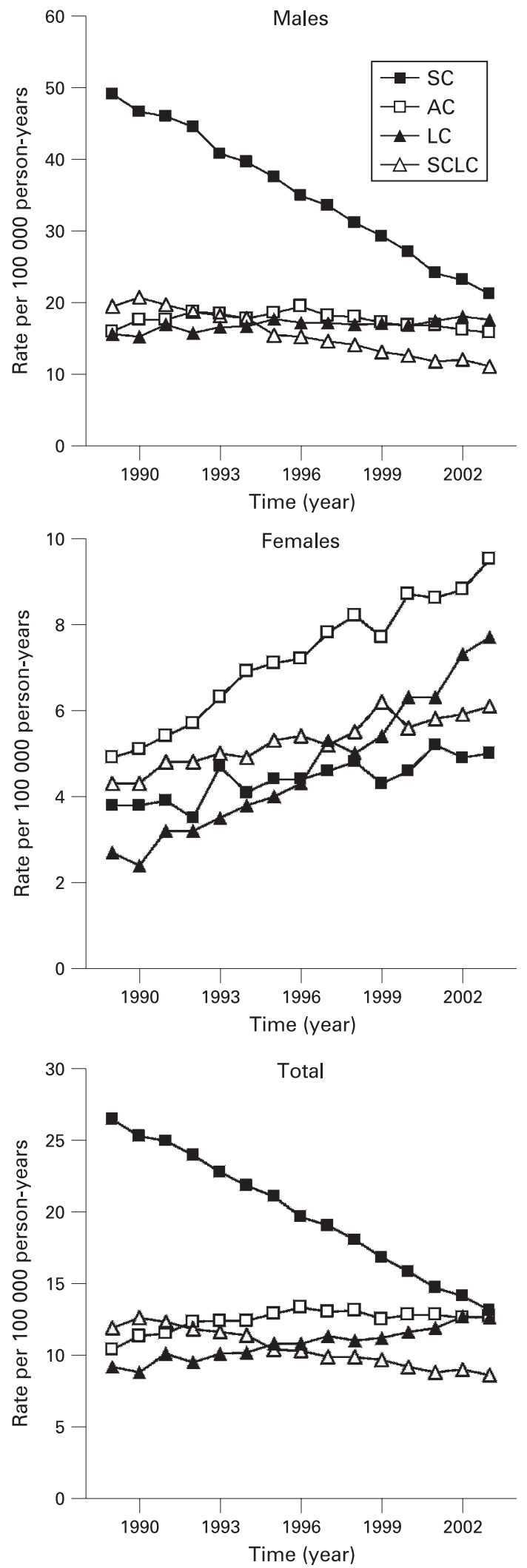

Figure 1 Trends in the incidence of squamous carcinoma (SC), adenocarcinoma (AC), large cell carcinoma (LC) and small cell lung cancer (SCLC) among men, women and both sexes combined in the Netherlands from 1989 to 2003 . The incidence is age-adjusted to the European Standard Population.

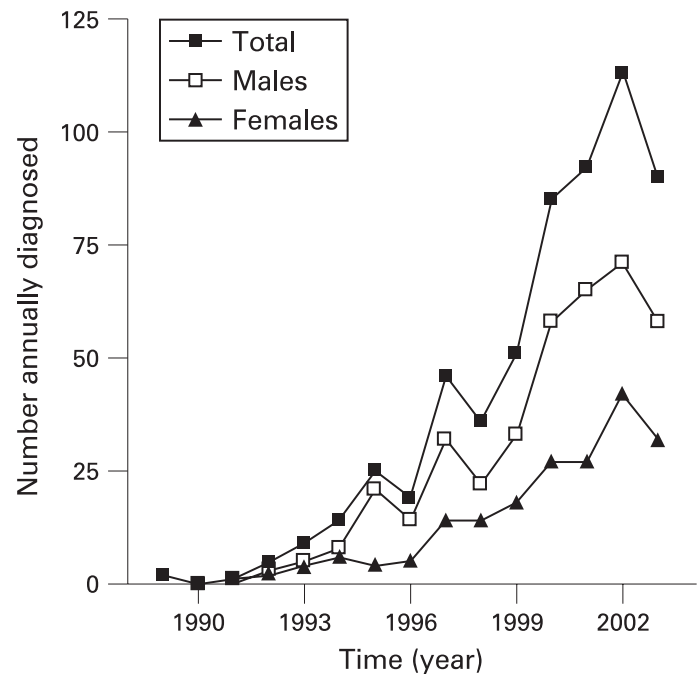

Figure 2 Number of large cell neuroendocrine carcinoma (LCNEC) diagnosed annually in the Netherlands. In 1999 a revision of the WHO classification of lung tumours was introduced ${ }^{22}$ with LCNEC from then on included as a separate entity in the spectrum of neuroendocrine tumours with non-small cell nuclear features.

\section{Adenosquamous carcinoma}

The most common subtype in the "other" group was adenosquamous carcinoma, accounting for $8.7 \%$ of all "other" tumours and $0.8 \%$ of all pulmonary tumours. The overall incidence of adenosquamous carcinoma decreased. This was caused by a decrease among men (EAPC $-5.7 \%$, CI $-7.3 \%$ to $-4.1 \%, p<0.0001)$; in women the incidence was constant.

\section{Carcinoid tumour}

The incidence of carcinoid tumours was constant at 0.44/ 100000 (approximately 70 each year in the Netherlands, $0.8 \%$ of all pulmonary tumours), with a male to female ratio of $48 \%$ to $52 \%$. Most carcinoid tumours ( $94 \%$ ) were classified as typical carcinoid. Nevertheless, the percentage of atypical carcinoid tumours is increasing (EAPC $+23.6 \%$, CI $14.3 \%$ to $33.6 \%$, $p=0.0001)$.

\section{Sarcomatoid carcinoma}

The incidence of sarcomatoid carcinomas (pleomorphic, spindle cell and giant cell carcinomas) remained constant at $0.17 /$ 100000 (approximately 25 annually, $0.3 \%$ of total). The male to female ratio of $75 \%$ to $25 \%$ also did not change. Among the subgroups of the sarcomatoid carcinomas, the number of spindle cell carcinomas increased at the cost of giant cell carcinomas in both sexes.

\section{Primary pulmonary sarcoma}

Finally, each year approximately 12 primary pulmonary sarcomas were diagnosed, with no change over time (annual incidence $0.08 / 100000$, EAPC $-0.6 \%$, CI $-3.4 \%$ to $2.3 \%$, $p=0.67)$. Primary pulmonary sarcomas were twice as common in men as in women.

\section{DISCUSSION}

During the 15-year study period over 134000 pulmonary tumours were diagnosed in the Netherlands. This very large number of well-defined tumours leads to a robust description of several important temporal trends. 

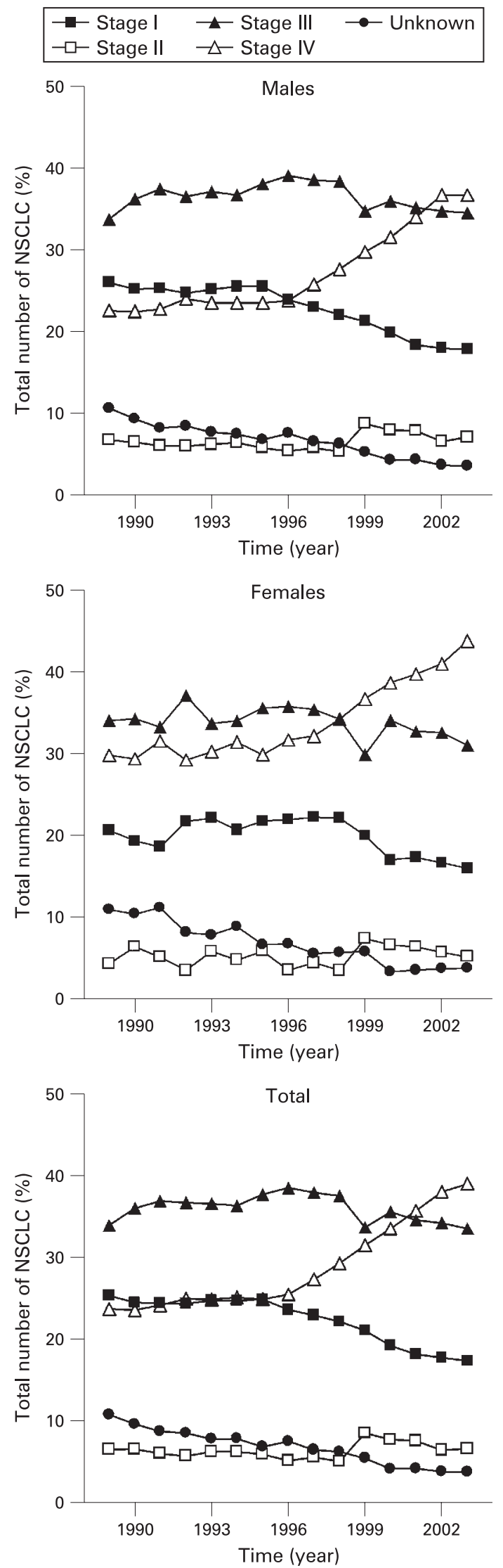

Figure 3 Stage distribution at diagnosis of non-small cell lung cancer (NSCLC) (squamous carcinoma, adenocarcinoma and large cell carcinoma) for men, women and both sexes combined.

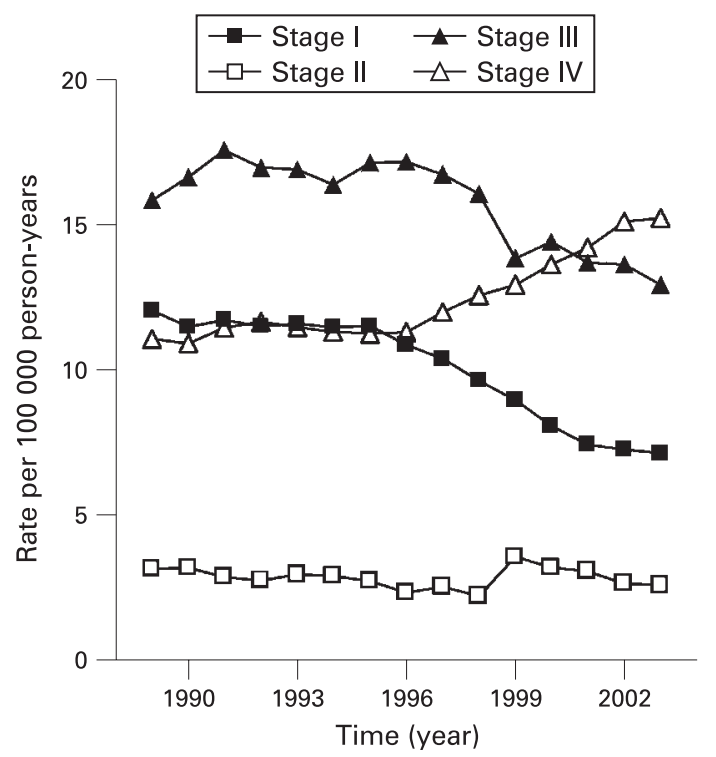

Figure 4 Trends in the incidence of the disease stage of non-small cell lung cancer (squamous carcinoma, adenocarcinoma and large cell carcinoma) in men and women combined in the Netherlands from 1989 to 2003. The incidence is age-adjusted to the European Standard Population.

The most remarkable finding in our study was that, after 1996, a sudden increase in the relative proportion of patients in stage IV was observed, with a concomitant decrease in patients in stage I. This trend seems to continue. It is unlikely that only patients in stage I are directly upstaged to stage IV; more plausible is that this upstaging occurs for all stages with more patients in stage IV in the end. One important reason for the stage shift is the introduction of positron emission tomography (PET) scanning. PET scanning was increasingly used in the Netherlands during the 1990s and became standard care in most hospitals around the year 2000. PET scanning results in the detection of previously occult distant metastasis, thereby upgrading patients with stage I, II or III disease to stage IV..$^{13}$ The availability of better quality CT scans has probably also resulted in upstaging. Apart from imaging, upstaging may be the result of the increase in the proportion of $A C$ because $A C$ is more prone to metastasise early in the course of the disease than the other subtypes of NSCLC. ${ }^{14}$ We observed that over $40 \%$ of patients with AC present in stage IV compared with only $25 \%$ of patients with SC. Moreover, women present with higher disease stages (for all subtypes) than men, and the number of women with lung cancer is increasing. Our results suggest that, currently, a higher proportion of patients is more accurately staged compared with a decade ago. This might have implications for the frequently used historical stage-specific survival tables for lung cancer ${ }^{15}$ which may represent an underestimation of stage-specific survival in the current times of modern staging techniques. Hypothetically, the observed stage shift could be reversed if CT-based lung cancer screening was implemented, which may lead to an increase in the number of patients in stage I and II.

Second, we observed that the overall incidence of lung cancer (SC, AC, LC and SCLC) in men is still decreasing (to 66/100 000 in 2003) whereas the increasing incidence in women (to 28.3/ 100000 in 2003) does not seem to be levelling off. The trends observed in the previous overview of lung cancer in the Netherlands $(1989-97)^{7}$ are therefore continuing. The main 

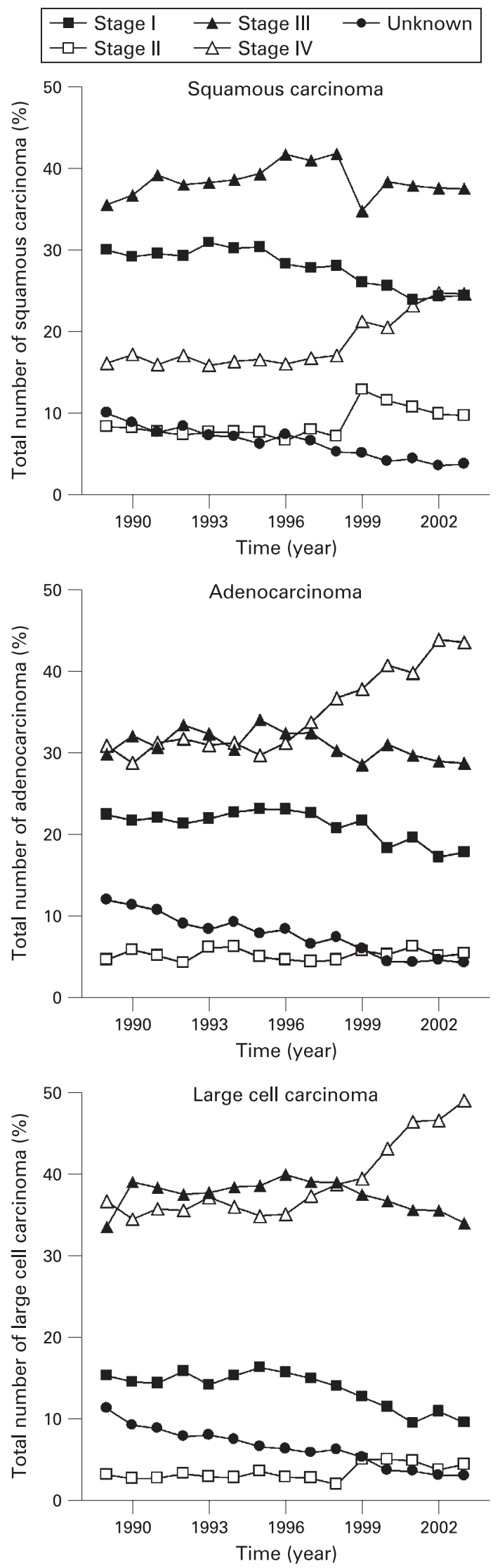

Figure 5 Stage distribution at diagnosis for squamous carcinoma, adenocarcinoma and large cell carcinoma (men and women combined).
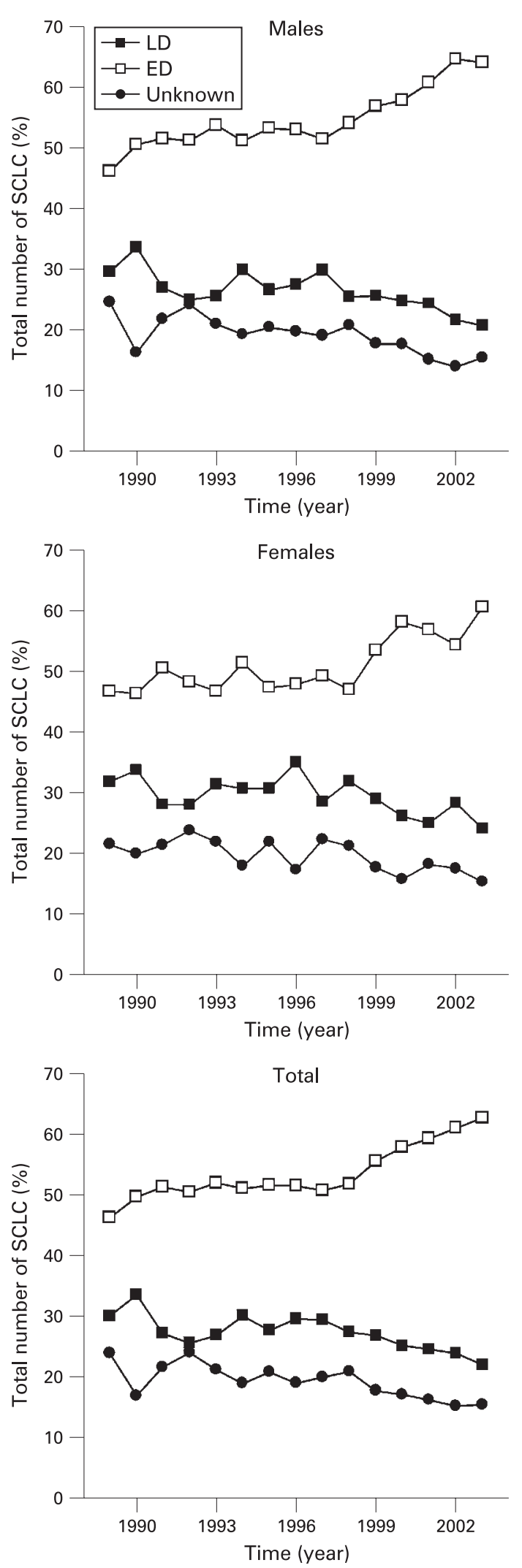

Figure 6 Stage distribution for small cell lung cancer (SCLC) at diagnosis for men, women and both sexes combined. ED, extensive disease; LD, limited disease. 
reason for these trends probably lies in the change in smoking habits. Since 1960 the percentage of men who smoked has decreased dramatically. In women the percentage who smoked increased in the 1950s and 1960s and thereafter decreased, albeit not so dramatically as in men. Because the time between the onset of smoking and the onset of lung cancer is relatively long, this decrease in smoking still has an effect. It is estimated that half of all lung cancers nowadays occur in former smokers. Currently, the incidence of lung cancer in the Netherlands is among the highest in Europe, but the highest incidence rates are observed in Eastern Europe. ${ }^{1}$ This is paralleled by the smoking rates, which are high in the Netherlands, but even higher in Eastern Europe. ${ }^{16} 17$

Several international studies have previously described changes in the epidemiology of NSCLC ${ }^{151819}$ and linked them to changes in (past) smoking rates. The temporal changes in incidence observed in the Netherlands occur more or less similarly throughout the Western World. These trends occur later in Eastern Europe because smoking cessation measures were started later. ${ }^{17}$ Because the decrease in smoking rates among men and the increase in smoking among women started earlier in the USA than in Europe, ${ }^{5}{ }^{19}$ the current incidence of lung cancer in the USA may reflect the future incidence in Europe in the coming decade.

Third, we observed that the distribution of the histological subtypes of lung cancer was significantly different between men and women and changed over time. In men, SC was formerly by far the most common subtype and, although the incidence has decreased, it is still the most common type in men followed by LC, AC and SCLC. In women all four subtypes increased, with $\mathrm{AC}$ being by far the most common type and SC the least common type. These changes could also be linked to changes in smoking habits. With less smoking, the number of tumours most strongly associated with smoking (SC and SCLC ${ }^{20}$ ) tend to decrease, whereas the number of lung cancers less strongly associated with smoking such as AC (also the most common subtype in lung cancer in never-smokers ${ }^{21}$ ) tend (relatively) to increase. Apart from the percentage of smokers, it is likely that the introduction of filter cigarettes-which were more popular among women than among men-is responsible for the relative increase in the proportion of $\mathrm{AC}$, especially among women. ${ }^{9}$ Filter cigarettes require deeper inhalation than non-filter cigarettes, causing a more peripheral particle deposition and therefore a predilection for peripherally occurring tumours (AC) instead of more central tumours (SC). In the Netherlands, filter cigarettes are still more popular than non-filter cigarettes. In $2003,73 \%$ of smoking men smoked normal filter cigarettes, $14 \%$ low-tar filter cigarettes and $11 \%$ non-filter cigarettes. In women these percentages were $66 \%, 29 \%$ and $4 \%$, respectively. ${ }^{8}$

Neuroendocrine neoplasms of the lung include (a)typical carcinoids, large cell neuroendocrine carcinoma (LCNEC) and SCLC, constituting a prognostic spectrum. ${ }^{22}{ }^{23}$ The fourth finding of our study was a steep increase after 1999 in the number of LCNEC. It is likely that this is because LCNEC were classified separately from SCLC in the 1999 revision of pathological classification. ${ }^{22}$ Also, the more frequent use of immunohistochemistry aimed at neural and endocrine markers could have caused the increasing number of neuroendocrine carcinomas. It is unknown whether this steep increase in the number of neuroendocrine carcinomas will continue. Carcinoid tumours constituted $0.8 \%$ of all pulmonary tumours, which is in line with other reports. ${ }^{24}{ }^{25}$ In our study the incidence of carcinoid tumours did not change $(0.44 / 100000$ for men and women together). Older studies suggest that the incidence of pulmonary carcinoid tumours is rising (study periods 1978-97 and 1973-91), ${ }^{25} 26$ although their observed maximal incidence is not higher than that of the present study (1989-2003). This rise in incidence in the 1970s and 1980s can be attributed to the better availability of fibreoptic bronchoscopy, so it is questionable whether this observed increase is accurate. Our observation that currently $10-15 \%$ of all carcinoid tumours are classified as atypical is in line with other studies..$^{23}$

Finally, our study focused on the less common pulmonary tumours and discerned, besides the aforementioned carcinoid tumours, three groups of uncommon tumours. Adenosquamous carcinomas, a rare subtype of NSCLC, constituted $0.8 \%$ of all pulmonary tumours, a little lower than the reported rate of 2.1$4.5 \%$ for resected lung cancers. ${ }^{28-30}$ One reason for the decrease in incidence of this smoking-related type of lung cancer with a poor prognosis probably lies in the decrease in smoking frequency. The sarcomatoid tumours are a heterogeneous group of smoking-related poorly differentiated aggressive tumours. ${ }^{31}$ With a decrease in smoking, a decrease in the incidence of these tumours is expected, but this was not observed probably because of the low overall numbers. The last subgroup of uncommon pulmonary tumours was the primary pulmonary sarcomas which represented approximately $0.1 \%$ of all pulmonary tumours, with a stable incidence. These rare tumours are not smoking-related and mostly comprise angiosarcoma, leiomyosarcoma and rhabdomyosarcoma..$^{32}$ No causal factor is known.

In conclusion, this large study showed an increase in the incidence of lung cancer in women and a significant decrease in the number of SC. AC is rapidly becoming the most common subtype. A remarkable stage shift was observed, with a still increasing proportion of stage IV disease at diagnosis which could be explained by the increased availability of PET scanning. The incidence of less common non-smoking-related pulmonary tumours did not change.

Funding: This study was supported by the Netherlands Cancer Registry (NCR). No specific funding was available for this study.

\section{Competing interests: None.}

Ethics approval: This study was approved by the National Cancer Registry (NCR) scientific committee and all procedures were performed according to NCR privacy regulations.

\section{REFERENCES}

1. Tyczynski JE, Bray F, Parkin DM. Lung cancer in Europe in 2000: epidemiology, prevention, and early detection. Lancet Oncol 2003;4:45-55.

2. Jemal A, Siegel R, Ward E, et al. Cancer statistics, 2007. CA Cancer J Clin 2007:57:43-66.

3. Parkin DM, Whelan SL, Ferlay J, et al. Cancer incidence in five continents. Vol VIII. Lyon, France: International Agency for Research on Cancer, 2002.

4. Doll $\mathbf{R}$, Hill $A B$. The mortality of doctors in relation to their smoking habits. $A$ preliminary report. BMJ 1954;228:1451-5.

5. Devesa SS, Bray F, Vizcaino AP, et al. International lung cancer trends by histologic type: male:female differences diminishing and adenocarcinoma rates rising. Int J Cancer 2005;117:294-9.

6. Forey $\mathbf{B}$, Hamling $\mathrm{J}$, Lee $\mathrm{P}$, et al. International smoking statistics: a collection of historical data from 30 economically developed countries. 2nd ed. Oxford: Oxford University Press, 2002

7. Janssen-Heijnen ML, van Dijck JA, Siesling S, et al. [Lung cancer in the Netherlands in the period 1989-1997: the epidemic is not over yet]. Ned Tijdschr Geneeskd 2001;145:419-23.

8. STIVORO. www.stivoro.nl (accessed 6 December 2007).

9. Thun MJ, Lally CA, Flannery JT, et al. Cigarette smoking and changes in the histopathology of lung cancer. J Natl Cancer Inst 1997;89:1580-6.

10. Post PN, Casparie MK, ten Kate FJ, et al. [The epidemiology of tumors of the testes in the Netherlands: accurate rendering by the Registry of Histopathology and Cytopathology (PALGA)]. Ned Tijdschr Geneeskd 2004;148:1150-4.

11. Fritz AG, Percy C, Jack A, et al. International Classification of Diseases for Oncology (ICD-0). 3rd ed. Geneva: World Health Organization, 2000. 
12. Statistics Netherlands (CBS). http://statline.cbs.nl/StatWeb/ laccessed 6 December 2007).

13. Pieterman RM, van Putten JW, Meuzelaar JJ, et al. Preoperative staging of nonsmall-cell lung cancer with positron-emission tomography. N Engl J Med 2000;343:254-61.

14. Shi AA, Digumarthy SR, Temel JS, et al. Does initial staging or tumor histology better identify asymptomatic brain metastases in patients with non-small cell lung cancer? J Thorac Oncol 2006;1:205-10.

15. Mountain CF. The international system for staging lung cancer. Semin Surg Oncol 2000; 18:106-15

16. Bray F, Tyczynski JE, Parkin DM. Going up or coming down? The changing phases of the lung cancer epidemic from 1967 to 1999 in the 15 European Union countries. Eur J Cancer 2004;40:96-125

17. Tyczynski JE, Bray F, Aareleid T, et al. Lung cancer mortality patterns in selected Central, Eastern and Southern European countries. Int J Cancer 2004;109:598-610.

18. Janssen-Heijnen ML, Coebergh JW. The changing epidemiology of lung cancer in Europe. Lung Cancer 2003;41:245-58.

19. Fu JB, Kau TY, Severson RK et al. Lung cancer in women: analysis of the national Surveillance, Epidemiology, and End Results database. Chest 2005;127:768-77.

20. Khuder SA. Effect of cigarette smoking on major histological types of lung cancer: a meta-analysis. Lung Cancer 2001;31:139-48.

21. Subramanian J, Govindan R. Lung cancer in never smokers: a review. J Clin Oncol 2007:25:561-70.

22. Travis WD, Colby TV, Corrin B, et al. World Health Organization international histological classification of tumors: histologic typing of lung and pleural tumors. 3rd ed. Berlin: Springer Verlag, 1999.
23. Asamura H, Kameya T, Matsuno $Y$, et al. Neuroendocrine neoplasms of the lung: a prognostic spectrum. J Clin Oncol 2006;24:70-6.

24. Hage R, de la Riviere $A B$, Seldenrijk CA, et al. Update in pulmonary carcinoid tumors: a review article. Ann Surg Oncol 2003;10:697-704.

25. Skuladottir H, Hirsch FR, Hansen HH, et al. Pulmonary neuroendocrine tumors: incidence and prognosis of histological subtypes. A population-based study in Denmark. Lung Cancer 2002;37:127-35.

26. Modlin IM, Sandor A. An analysis of 8305 cases of carcinoid tumors. Cancer 1997; 79:813-29.

27. Garcia-Yuste M, Matilla JM, Cueto A, et al. Typical and atypical carcinoid tumours: analysis of the experience of the Spanish Multi-centric Study of Neuroendocrine Tumours of the Lung. Eur J Cardiothorac Surg 2007;31:192-7.

28. Gawrychowski J, Brulinski K, Malinowski E, et al. Prognosis and survival after radical resection of primary adenosquamous lung carcinoma. Eur J Cardiothorac Surg 2005:27:686-92.

29. Kang SM, Kang HJ, Shin JH, et al. Identical epidermal growth factor receptor mutations in adenocarcinomatous and squamous cell carcinomatous components of adenosquamous carcinoma of the lung. Cancer 2007;109:581-7.

30. Nakagawa K, Yasumitu T, Fukuhara K, et al. Poor prognosis after lung resection for patients with adenosquamous carcinoma of the lung. Ann Thorac Surg 2003:75:1740-4.

31. Rossi G, Cavazza A, Sturm N, et al. Pulmonary carcinomas with pleomorphic, sarcomatoid, or sarcomatous elements: a clinicopathologic and immunohistochemical study of 75 cases. Am J Surg Pathol 2003;27:311-24.

32. Gladish GW, Sabloff BM, Munden RF, et al. Primary thoracic sarcomas. Radiographics 2002:22:621-37.

\section{Lung alert}

\section{Increasing incidence of pulmonary embolism}

The introduction of investigations like spiral CT scanning and D-dimer assay have altered the outcome of patients with pulmonary embolism in recent years. This study aimed to determine whether there had been a significant improvement in the diagnosis of pulmonary embolism and, consequently, a reduction in mortality with the advent of spiral CT.

The study population consisted of inpatients in Pennsylvania, USA between 1997 and 2001. Pregnant patients and those with pulmonary embolism admitted for the second time or later were excluded. The authors calculated the incidence of pulmonary embolism per year, population risk, proportion of patients undergoing scanning and inhospital mortality. The incidence of pulmonary embolism as well as population risk increased steadily from 1997 to 2001, with a mean increase of $0.004 \%$ per year for the former and $0.008 \%$ for the latter $(\mathrm{p}<0.001$ for both). There was also a significant increase in the proportion of patients undergoing CT scanning, particularly spiral CT, which accounted for $71.2 \%$ of the scans in 2001 compared with $30.3 \%$ in 1997. The proportion of inhospital deaths was significantly lower in 2000 and subsequently than in the years up to and including 1999.

This study has highlighted the possibility of a rising incidence of pulmonary embolism due to spiral CT scanning leading to the increased diagnosis of small emboli-many of unclear significance. However, it is difficult to assess the role of other factors such as changes in physician index of suspicion. There is a need for further studies to look specifically at the relevance of such emboli and their long-term outcome.

- DeMonaco NA, Dang 0, Kapoor WN, et al. Pulmonary embolism incidence is increasing with use of spiral computed tomography. Am J Med 2008;121:611-7

\section{J Mathew}

Correspondence to: Dr J Mathew, LAT SpR, Colchester General Hospital, Colchester, UK; jovin mathew@yahoo.com 